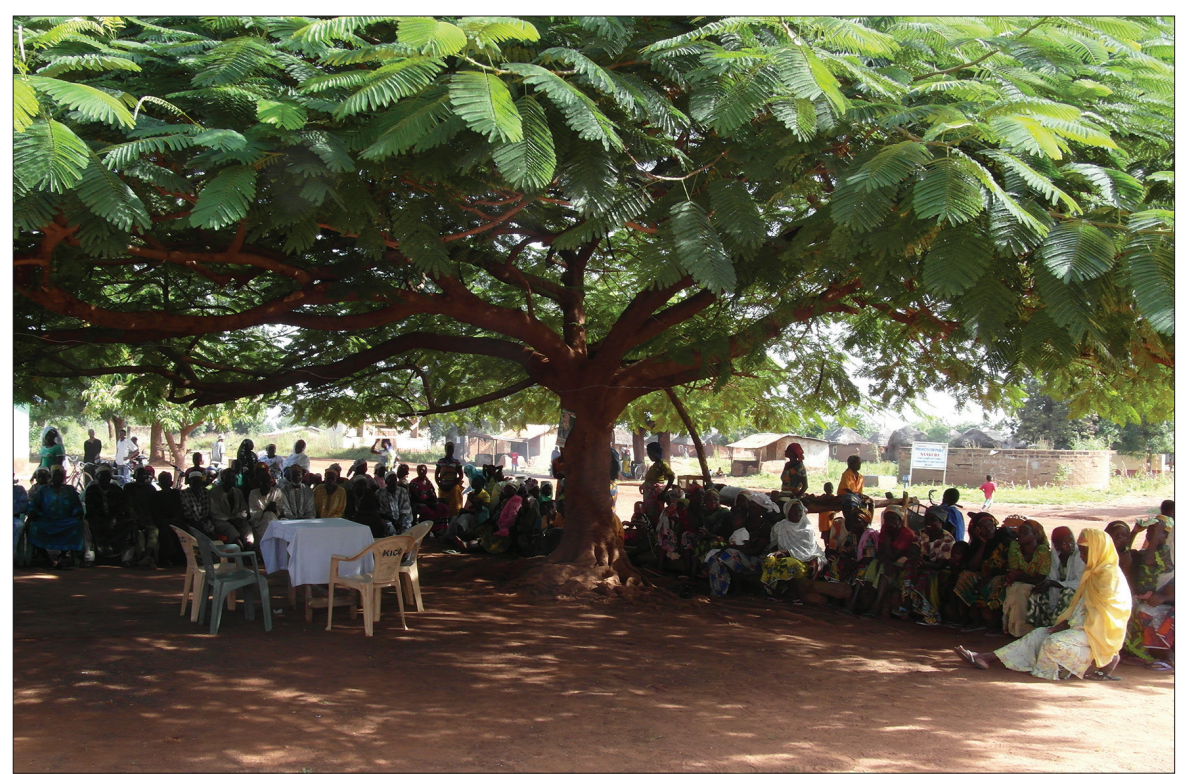

Waiting to see the doctor in Tamale, Northern Ghana.

electronic system which is marginally used and not regularly updated. Overall, primary care in Slovenia is under-funded.

\section{Jenny Katsoni,}

GP ST2, East Sussex GP training scheme

Eastbourne and Hastings.

Email: jkatsonidgmail.com

\section{GHANA}

In October 2012 | travelled to Ghana for 12 days with a team of 10 other health professionals with the organisation Evangelistic Medical Missions Abroad. We spent 4 days working in medical outreach clinics in rural villages near Tamale in the Northern Region of Ghana and 1 day working alongside the staff at a local mission hospital, The King's Medical Centre.
In those few days we saw over 1200 patients with a huge variety of medical conditions, including malaria, gastroenteritis and dehydration, musculoskeletal complaints, and skin diseases. Imagine arriving at work in the morning to set up your "consultation room' under the shade of a tree in the middle of a dusty village with a queue of over 100 people already waiting to see you.

Non-specific complaints are not confined to western culture: a number of patients complained of all-over body pain'. It was extremely difficult to try and get an accurate time frame of somebody's presenting complaint: asking a patient to specify what they meant by 'a long time' often just resulted in the response of 'a very long time'. A number of patients presented very late either with severe acute conditions or with problems which had been ongoing for many years. One of the biggest challenges was knowing how to manage patients with chronic conditions such as hypertension: is it worth giving somebody a 2-week supply of antihypertensives knowing that the probability is that they will not get any more after this? For patients with 'red flag' symptoms investigations such as CT scans and colonoscopies were virtually inaccessible, let alone 2-week wait referrals.

I vividly remember a young boy who attended with his parents who reported that he was 14 years old but weighed just 18 kilograms. He was unable to communicate and had been like this since a febrile illness when 3 years old; presumably some sort of cerebral infection. He was clearly in need of intensive therapy but these services are largely unavailable in Ghana. Trying to explain this to the parents and the limitations of the help you could give was difficult. The skills I developed in complex communication, dealing with a diverse disease burden, and managing large numbers of patients in the community, will only contribute to my training as a GP

\section{Rebecca Hancock,}

GP ST1 Bournemouth.

Email: beckyhancock@doctors.org.uk

DOI: 10.3399/bjgp13X674549

\section{Further information}

http://vdgm.woncaeurope.org/content/abouthippokrates
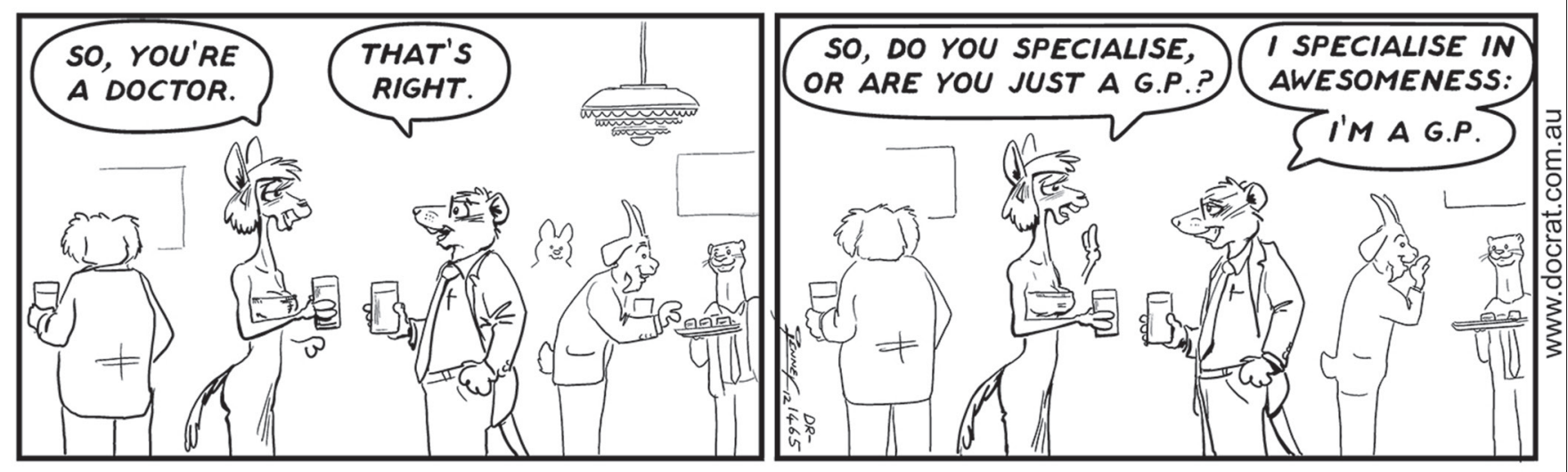